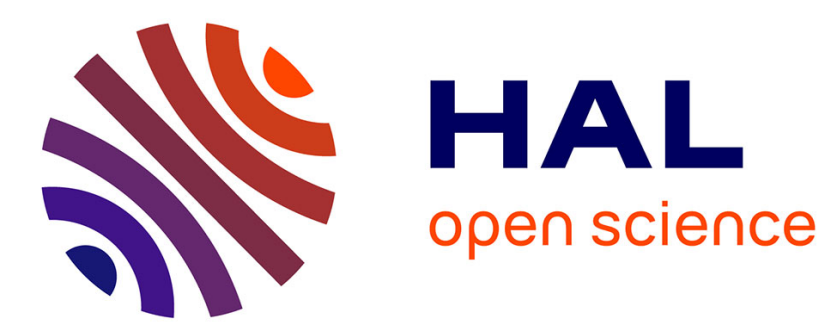

\title{
Automated visual inspection of an airplane exterior
}

\author{
Igor Jovančević, Jean-José Orteu, Thierry Sentenac, Rémi Gilblas
}

\section{To cite this version:}

Igor Jovančević, Jean-José Orteu, Thierry Sentenac, Rémi Gilblas. Automated visual inspection of an airplane exterior. 12th International Conference on Quality Control by Artificial Vision (QCAV'2015), Jun 2015, Le Creusot, France. 10.1117/12.2182811 . hal-01351735

\section{HAL Id: hal-01351735 \\ https://hal.science/hal-01351735}

Submitted on 4 Aug 2016

HAL is a multi-disciplinary open access archive for the deposit and dissemination of scientific research documents, whether they are published or not. The documents may come from teaching and research institutions in France or abroad, or from public or private research centers.
L'archive ouverte pluridisciplinaire HAL, est destinée au dépôt et à la diffusion de documents scientifiques de niveau recherche, publiés ou non, émanant des établissements d'enseignement et de recherche français ou étrangers, des laboratoires publics ou privés. 


\title{
Automated visual inspection of an airplane exterior
}

\author{
Igor Jovančević, Jean-José Orteu, Thierry Sentenac and Rémi Gilblas \\ Université de Toulouse ; INSA, UPS, Mines Albi, ISAE ; ICA (Institut Clément Ader) ; \\ Campus Jarlard, F-81013 Albi, France
}

\begin{abstract}
This paper deals with the inspection of an airplane using a Pan-Tilt-Zoom camera mounted on a mobile robot moving around the airplane. We present image processing methods for detection and inspection of four different types of items on the airplane exterior. Our detection approach is focused on the regular shapes such as rounded corner rectangles and ellipses, while inspection relies on clues such as uniformity of isolated image regions, convexity of segmented shapes and periodicity of the image intensity signal. The initial results are promising and demonstrate the feasibility of the envisioned robotic system.
\end{abstract}

Keywords: inspection, airplane exterior, image processing, PTZ camera

\section{INTRODUCTION}

Airplanes are periodically inspected, either on the airport tarmac between two fligths or in a hangar during maintenance operations. Today, these inspections are done by human operators, mainly visually and sometimes with the help of some inspection tools, for instance to evaluate the criticality of a crack detected on the plane fuselage. In order to make the inspection quicker, more exhaustive and more accurate, as well as for traceability reasons, a multi-partners research project is being carried on in order to develop a mobile robot equipped with several optical sensors (cameras and 3D scanners) to perform an automatic inspection of an airplane.

The approach chosen in the project is based on an autonomous mobile robot on the ground, capable to communicate with human operators and infrastructures around the aircraft. The collaborative robot (cobot) has to move autonomously between different checkpoints while interacting with pilot, workers, vehicles and airport information systems. In addition to the long range navigation task, at each checkpoint (around twenty for a preflight inspection) the cobot has to perform an inspection task of several items (probes, doors, etc.). Around 240 items to be inspected are identified for the whole plane. Automated image acquisition is provided by controlling sensors and pointing them towards the item to be inspected. This is feasible by taking into account the position of the robot with respect to the plane and the position of the item in the 3D model of the plane. Inspection is then performed by processing the Pan-Tilt-Zoom (PTZ) camera images and 3D point clouds provided by a 3D scanner. For each item, integrity or right configuration should be verified. Accordingly, one of the three general strategies is then chosen and applied: $(\boldsymbol{A})$ single view $2 D$ image processing; $(\boldsymbol{B})$ model based image processing (single view image processing by using 3D CAD model of the element); $(\boldsymbol{C})$ processing of $3 D$ point clouds. First strategy is preferred whenever possible, for the time reasons. Second strategy is used when image processing is not sufficient, for ex. for inspecting probes. Third strategy is employed for detecting damages on the airplane skin (cracks, dents, bumps).

Robotic airplane inspection from the ground, based on computer vision, is a specific application not yet studied. Previous efforts ${ }^{1-5}$ were mainly focused on detailed airplane skin inspection with robot crawling on the airplane surface. In most cases, industrial automation systems ${ }^{6}$ are used to inspect known objects at fixed positions with appropriate illumination necessary to extract meaningful features. In our application, however, sensors are mounted on the moving robot and no additional illumination is used. Moreover, our intention is to enable robot to cover as many items as possible from one position, i.e. to move as less as possible. Since 2D image processing approaches are preferred, initial strategy is to assess performances of the 2D image processing methods and this article is focused on presenting these results.

E-mail: igor.jovancevic@mines-albi.fr

Twelfth International Conference on Quality Control by Artificial Vision, edited by

Fabrice Meriaudeau, Olivier Aubreton, Proc. of SPIE Vol. 9534, 95340Y · C 2015

SPIE · CCC code: $0277-786 X / 15 / \$ 18 \cdot$ doi: $10.1117 / 12.2182811$

Proc. of SPIE Vol. 9534 95340Y-1 


\section{PROPOSED METHODS FOR FOUR TYPES OF ITEMS}

Results in inspecting some of the items will be presented. For all the items, the approach comprises detection of region of interest (ROI) and the inspection, i.e. making decision. When possible, the presence of other detectable elements is exploited to narrow down the search area for an item to be inspected. Adopted strategy is to detect the ROI, point the camera in order to have the item in the center of the image, zoom in and finally perform the inspection on the zoomed item. Since the inspection is done only on zoomed images, we can accept certain level of imprecision in ROI detection on small zoom images.

Usual weakness of an inspection system is being specialized in inspecting one particular object. In that sense, each of the items on the plane could be an isolated, specific, inspection problem. Our intention is to design more general strategies, applicable to a class of similar items by changing just parameters and geometry. Methods are tested on a dataset of real A320 airplane images acquired by PTZ camera in AIRBUS hangar (Fig. 1).

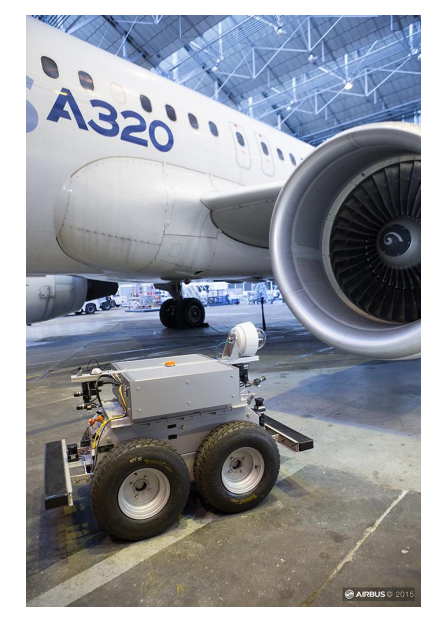

Figure 1. Robot in the hangar (Courtesy of AIRBUS).

\subsection{Preprocessing step: background removal}

When the robot is placed on one of the sides of the plane (Fig. 2a), complex structures behind the plane, such as hangar construction, are eliminated as highly textured areas close to the image borders. This way we are keeping middle, mainly uniform part of the image which corresponds to the airplane surface. To enhance textured regions, the original, grayscale, smoothed image is convolved with $5 \times 5$ Laws kernel mask ${ }^{7}$ obtained by multiplying two one-dimensional vectors:

$$
\left[\begin{array}{c}
1 \\
-4 \\
6 \\
-4 \\
1
\end{array}\right] \times\left[\begin{array}{lllll}
-1 & 0 & 2 & 0 & -1
\end{array}\right]
$$

On the resulting image, windowing operation of neighborhood averaging followed by morphological dilation and contrast enhancement is then applied (Fig. 2b). Further, region growing segmentation is performed on the texture image (Fig. 2c). For locating the border between the plane and the background, we rely on the fact that this border is a straight line and that the airplane is in the upper part of the image. By employing the Random Sample Consensus technique ${ }^{8}$ for removing outliers, we are fitting a line to the points obtained as peaks of the regions emerging from lower border of the image (Fig. 2d). Finally, image region below the fitted line is eliminated in further processing. 


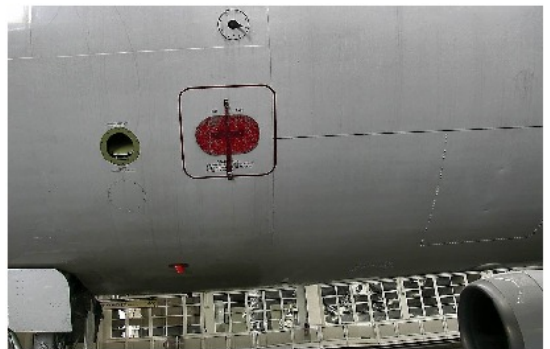

(a) Original image.

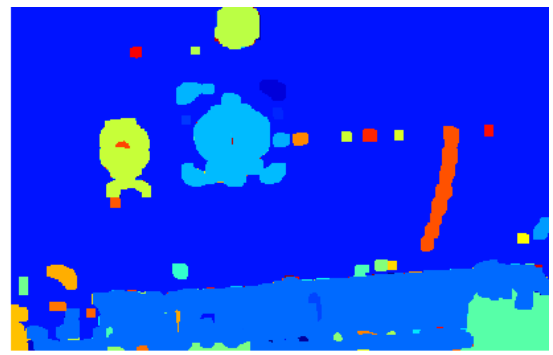

(c) Region growing segmentation on texture image.

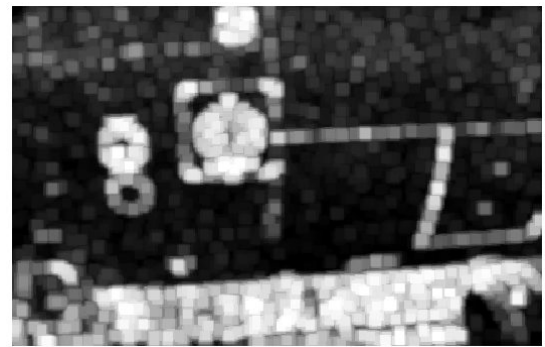

(b) Texture image after dilation and contrast enhancement.

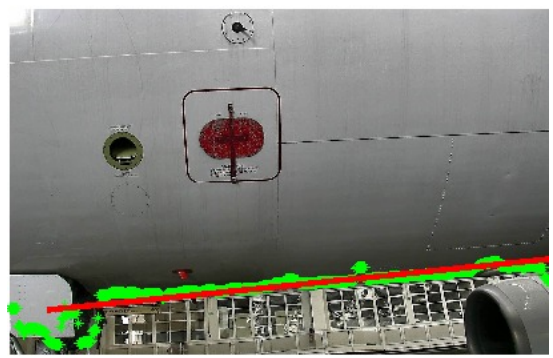

(d) Line fitted on the points which are on the top of the regions emerging from the low border of the image.

Figure 2. Removing the background.

\subsection{Oxygen bay handle}

Objective. The objective is to check if the handle is latched (Fig. 3). The problem of ROI detection is to a certain degree similar to the problem of edge based door detection ${ }^{9-12}$ with monocular camera. Neither of the cited methods is fully applicable to our problem. Ref. 9 relies on corners and our corners are rounded, while Refs. 10, 11 and 12 do not enable rotational invariance of a door.

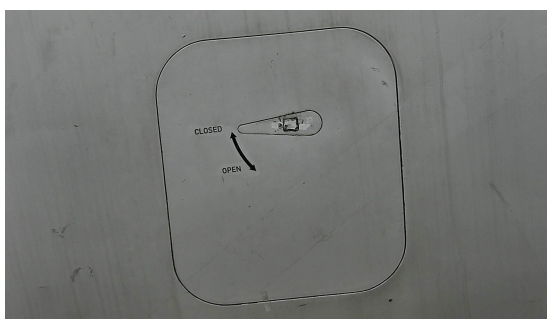

(a) Latched Oxygen bay (NEGATIVE).

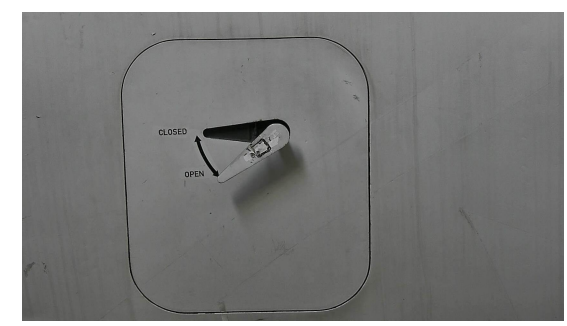

(b) Unlatched Oxygen bay (POSITIVE).

Figure 3. Oxygen bay - negative and positive example.

ROI detection. On the edge image, we apply Hough Transform $(\mathrm{HT})^{13}$ in order to detect the straight lines. HT suffers from computational complexity but in our case the edge set is not large. Since the scene can be complex, we allow detection of many lines, in order not to miss some of the lines we are looking for. Among 
many detected lines, the challenge is to pick four lines which bound the Oxygen Bay. Firstly, many four lines (two pairs) candidates are selected such that within each pair two lines are close to parallel and between two pairs lines are far from parallel (ideally perpendicular). We can safely assume that perspective distortion of the airplane surface is negligible, i.e. we can count to a certain degree on affine invariants such as parallelism of lines. Weakness of the HT is that it returns rays and not continuous segments, neglecting the gaps between parts of a ray. Therefore, it can happen that we obtain a ray which is constituted by two or more distant segments (Fig. 4b). To avoid this, once the four rays and their intersections are detected, segments which correspond to rectangle sides are checked for fill ratio measure similarly to Ref. 9. Fill ratio of a segment is a measure of how much of the segment is supported by edge pixels. We are interested only in candidates whose all four segments are well supported by the edges (Fig. 4a) and we are rejecting those whose segments have big gaps (Fig. 4b). After fill ratio criteria is verified, the knowledge about real world aspect ratio of the sides of the rectangle is used

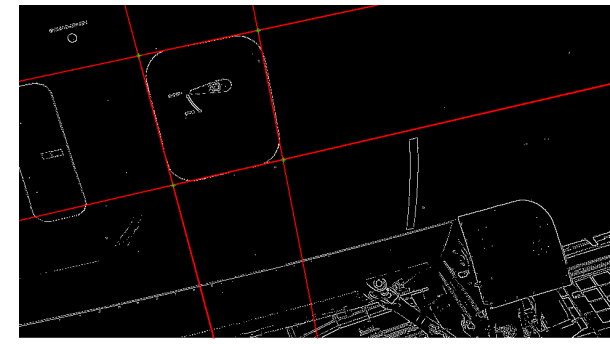

(a) Good candidate for being the Oxygen bay - all four segments are well supported by the edges.

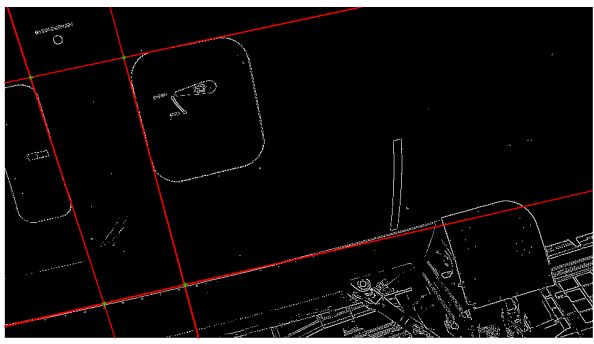

(b) Bad candidate for being the Oxygen bay - three out of four segments are poorly supported by the edges.

Figure 4. Two cases of four segment candidate (red) and their supporting edges (white).

to find the candidate whose sides are closest to this ratio. Finally, region of interest (ROI) is extracted as an area bordered by the four lines (Fig. 4a).

Inspection. The smaller ROI (handle) is further isolated as a convex hull of the set of edge points in the upper half of the big ROI (Fig. 5a and 5c). This small ROI is binarized by using Otsu method ${ }^{14}$ for adaptive thresholding (Fig. 5b and 5d). A clue used for making decision (LATCHED/UNLATCHED) is the ratio between the area of the black region and the area of the whole ROI. It is low in LATCHED case (Fig. 5b) and high in UNLATCHED case (Fig. 5d).

Evaluation. The detection approach was successful in the $96 \%$ of the cases on a dataset of 128 images of four different kinds of rectangular doors with different dimensions. The inspection method has shown the accuracy with $0 \%$ false negative rate (FNR) and $4.6 \%$ false positive rate (FPR) on the dataset of 122 images of four different kinds of doors each having different position of the handle.

\subsection{Air Inlet Vent}

Objective. The objective is to verify if the vent is closed (Fig. 6). Challenging case is when the inlet is partially open.

ROI detection. ROI detection is a known image processing problem of ellipse detection from the edge image. On the edge image we apply computationally efficient version of HT method ${ }^{15}$ for ellipse detection. 


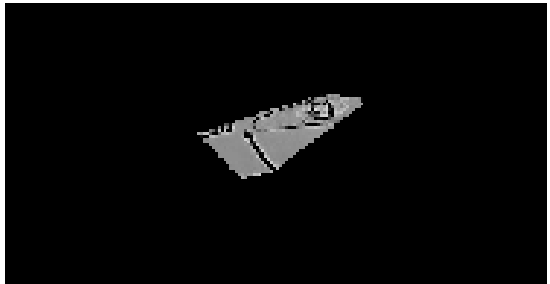

(a) LATCHED case: ROI.

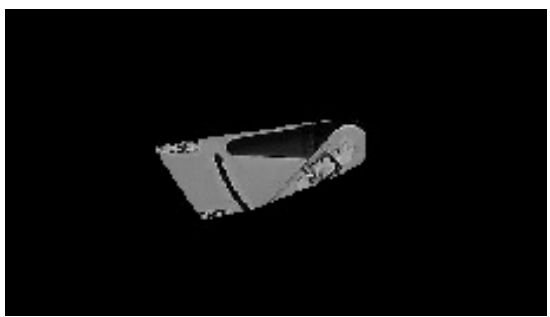

(c) UNLATCHED case: ROI.

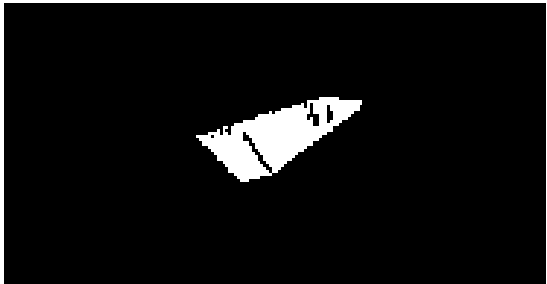

(b) LATCHED case: Binarized ROI. Black region is small with respect to the ROI.

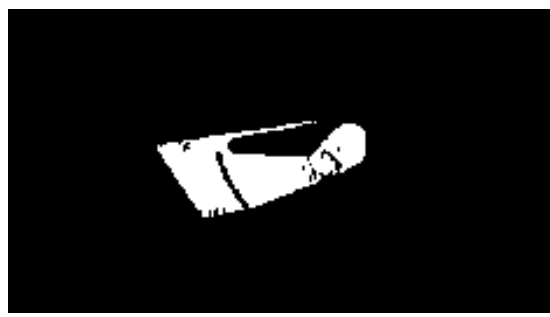

(d) UNLATCHED case: Binarized ROI. Black region is large with respect to the ROI.

Figure 5. Oxygen bay - ROI and binarized ROI.

Inspection. In order to perform inspection, we could estimate the binarized vent region similarly as we did with the handle in Sec. 2.2. However, since the vent can be only partially open, binarization result of the whole ROI could be ambiguous as a clue. Thereby, for a more detailed inspection, a small sliding window is moved along the border of ROI (Fig. 6) and uniformity of the sliding window is estimated by the binarization. In the

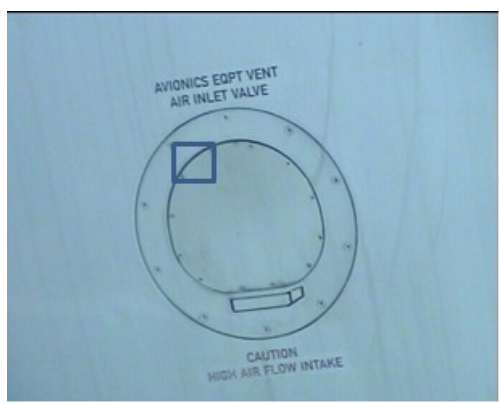

(a) CLOSED vent (NEGATIVE).

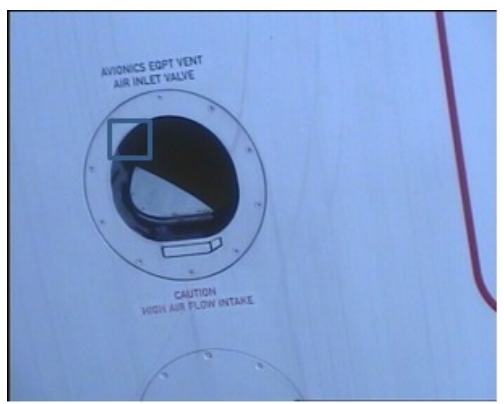

(b) OPEN vent (POSITIVE).

Figure 6. Air Inlet Vent - sliding window inspection.

case of OPEN vent, there are two different regions within sliding window, while in the case of CLOSED vent, the sliding window is uniform.

Evaluation. On the dataset of 23 low zoom images (ex. Fig. 2a), ROI detection approach has shown $95 \%$ accuracy. Inspection is performed with both FNR and FPR equal to $0 \%$, after a test on 32 high zoom images (ex. Fig. 6) of two different kinds of air inlet vent. 


\subsection{Static ports}

Objective. We need to verify that the protecting cover is removed from the static ports (Fig. 7).

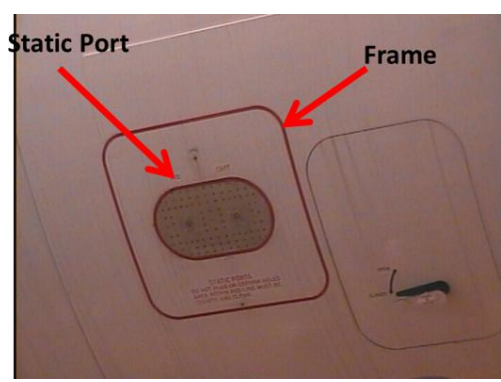

(a) Uncovered (NEGATIVE).

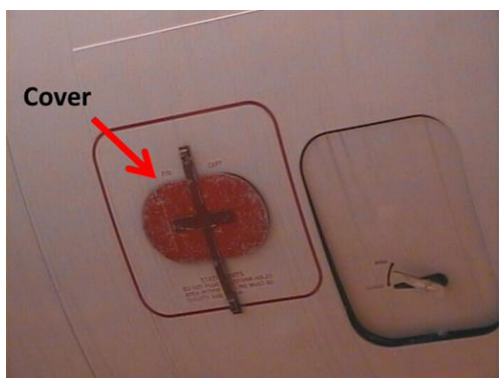

(b) Covered (POSITIVE).

Figure 7. F/O and CAPT static ports. Wrong situation is when protecting cover is still present (Fig. 7b).

ROI detection. Since there are red regions on the image, original RGB image is converted to CIELAB color space. A* channel of this color space is suitable for segmenting red regions. Further, from the input edge set, only edges corresponding to red regions are kept. The improvement can be seen in the Fig. 8. On the reduced

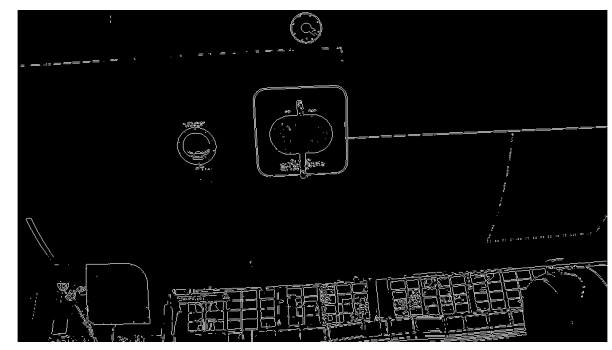

(a) All the edges of the input image.

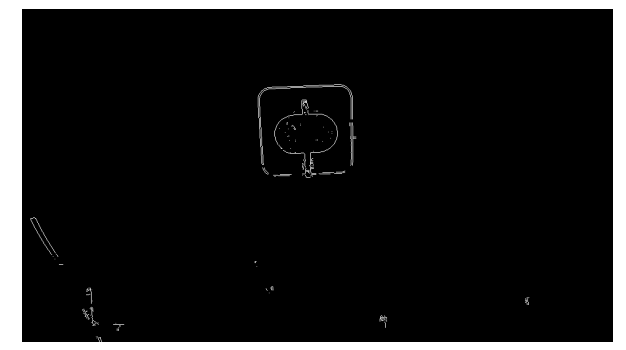

(b) Edges corresponding to red regions.

Figure 8. Significant reduction of edge set by rejecting edges of non-red regions.

set of edges (Fig. 8b), the approach explained in Sec. 2.2 is employed to detect rectangular ROI. Further, Active contours method ${ }^{16}$ is run within ROI for segmenting the static ports region.

Inspection. $\mathrm{A}^{*}$ channel is used as a clue for making a decision as well. Namely, mean grey level values of the regions shown in the Fig. 9a and $9 \mathrm{~b}$ are very close to each other (UNCOVERED case) while in the case shown in the Fig. 9c and 9d (COVERED case), they are different. Another clue is convexity of the detected static port region which is convex in UNCOVERED case (Fig. 9a) and concave in COVERED case (Fig. 9c).

Evaluation. The detection approach was successful in the $97 \%$ of the cases on the dataset of 98 images of two different kinds of static ports. The inspection method has shown accuracy with both FNR and FPR equal to $0 \%$, on the dataset of 96 images with two different cover shapes. 


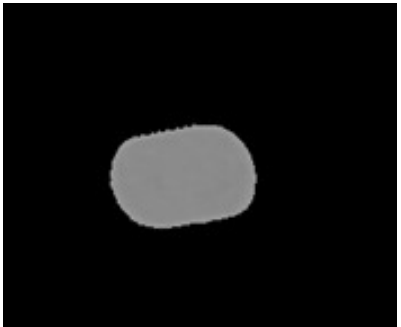

(a) UNCOVERED case: static ports region.

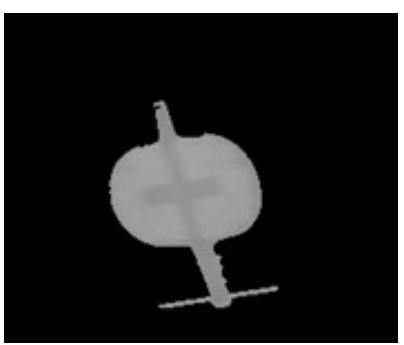

(c) COVERED case: static ports region.

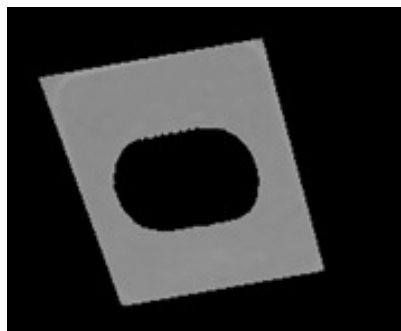

(b) UNCOVERED case: frame without static ports region.

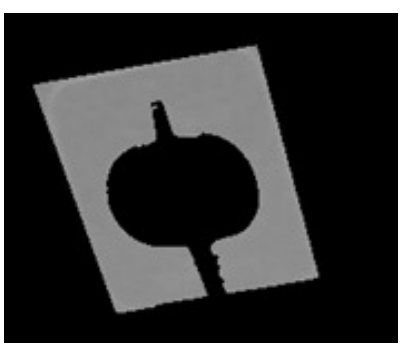

(d) COVERED case: frame without static ports region.

Figure 9. Static ports - UNCOVERED: both regions are not red; COVERED: region 9c is red, region 9d is not red.

\subsection{Fan blades}

Objective. In the blades region we should detect a large defect such as presence of visible object (Fig. 11a) or big damage like missing blade. For this item, we evaluate inspection approach by assuming ROI is already detected and the engine is zoomed.

Inspection. Grayscale intensity image is treated with histogram equalization technique for contrast enhancement because blades are usually not well illuminated being placed deep inside the engine inlet. Further, we extract pixel values of many circles from blades region (green in Fig. 12). Each circle is one dimensional signal which is extracted from the image and smoothed with Gaussian filter in order to eliminate small details. When there is no defect, a signal close to periodic is obtained (Fig. 10b). However, in the case of defect, there is usually a breach of this periodicity (Fig. 11b). By Fourier analysis we compute frequency of the highest magnitude component within the sliding window moved along the $1 \mathrm{D}$ signal. In the favorable case, this frequency is constant as the sliding window is moved. In the case when a defect is present, frequency is changed when the sliding window is in the region of the defect. Nevertheless, some false alarms are present in the cases of not illuminated regions and in the regions where details behind the blades are visible (Fig. 12b). To avoid these false alarms, only defect which is present on many consecutive circles is reported as a foreign object or a damage (Fig. 12a). For this reason, in this moment, the method is able to detect only large defects.

Evaluation. On the dataset of 49 images, the approach performed with $0 \% \mathrm{FNR}$ and $16 \% \mathrm{FPR}$. It failed in the cases with blades being poorly illuminated and not zoomed enough. The method should be improved with detection phase planned to precede the inspection and enable zooming the blades region for better inspection accuracy. 


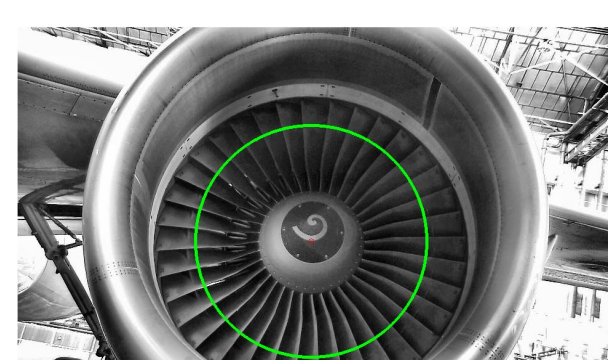

(a) Extracted circle. No defect.

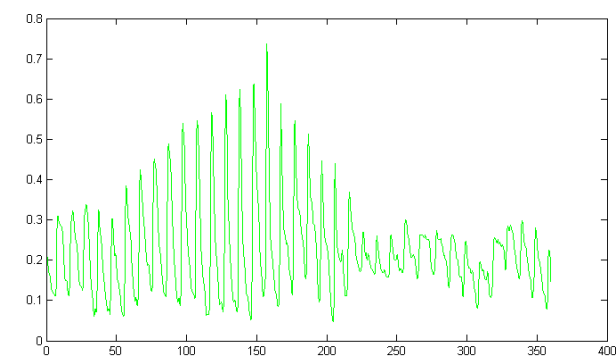

(b) Corresponding 1D signal of pixel intensities on the circle.

Figure 10. Example of 1D signal read from the input image with no defect.

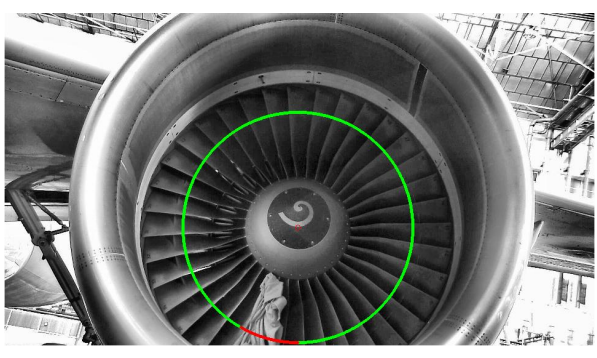

(a) Extracted circle. Red part is reported as defected.

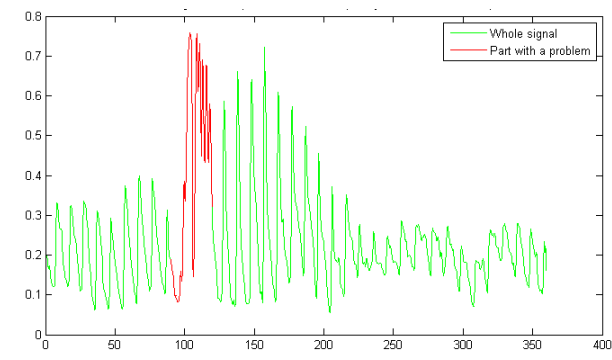

(b) Corresponding 1D signal of pixel intensities on the circle with red part corresponding to the defect.

Figure 11. Example of 1D signal read from the input image with defect.

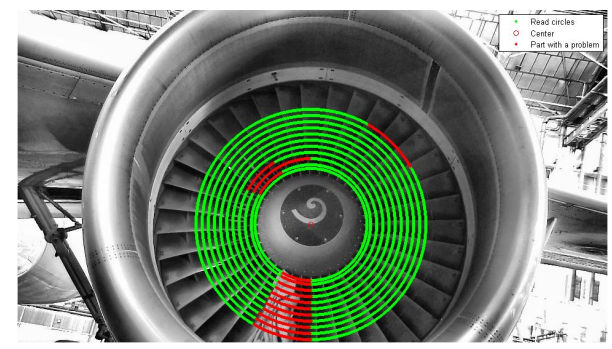

(a) Defect - present object.

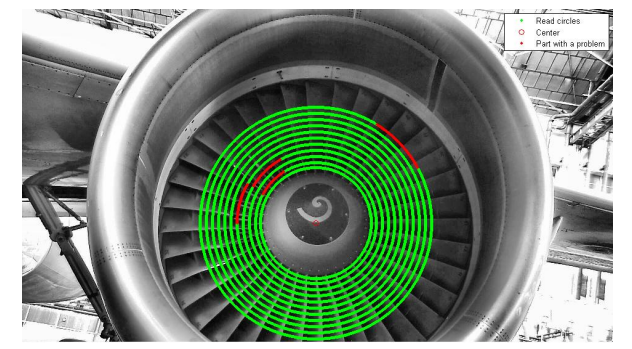

(b) No defect.

Figure 12. Fan blades - two cases to be distinguished and circular pixel sets which are analyzed. GREEN: circles which are extracted from the image; RED: parts where the approach reported a defect, i.e. breach of periodicity.

\section{CONCLUSION}

Several image processing methods, each solving a specific airplane inspection problem, were developed and evaluated on the real airplane exterior images. Detection approaches for three types of items showed accuracy of at least 95\%, while inspection approaches could reach 0\% FNR with acceptable false alarm rates (FPR). In general, whole process depends on detection phase which has been shown as more challenging than the inspection task. Since detection is performed on small zoom images, knowledge about presence of more than one item is integrated and sets of neighboring items are detected instead of detecting one particular item. Notably, 
our method for red regions detection has been shown quite robust, therefore it is often employed to facilitate detection of other items. In the article, we presented inspection approaches for four representative types of items. Nevertheless, other items marked as highly important by human inspectors, has been treated as well.

\section{ACKNOWLEDGMENTS}

This work is part of the AIR-COBOT project (http://aircobot.akka.eu) approved by the Aerospace Valley world competitiveness cluster. We gratefully acknowledge financial support from the French Government via the Single Inter-Ministry Fund (FUI). The partners of the AIR-COBOT project (AKKA TECHNOLOGIES, AIRBUS Group, ARMINES, 2MoRO Solutions, LAAS-CNRS, M3 SYSTEMS and STERELA) are also gratefully acknowledged for their support.

\section{REFERENCES}

[1] Siegel, M., Gunatilake, P., and Podnar, G., "Robotic assistants for aircraft inspectors," Instrumentation Measurement Magazine, IEEE 1, 16-30 (Mar 1998).

[2] Siegel, M., Kaufman, W., and Alberts, C., "Mobile robots for difficult measurements in difficult environments: Application to aging aircraft inspection," Robotics and Autonomous Systems 11(3-4), 187 - 194 (1993).

[3] Davis, I. L. and Siegel, M., "Automated nondestructive inspector of aging aircraft," in [Measurement Technology and Intelligent Instruments], Zhu, L., ed., Proc. SPIE 2101, 190-201, SPIE, Wuhan, China (1993).

[4] Gunatilake, P. and Siegel, M., "Remote enhanced visual inspection of aircraft by a mobile robot," in [Proc. 1998 IEEE Int'l Workshop on Emerging Technologies, Intelligent Measurement and Virtual Systems for Instrumentation and Measurement (ETIMVIS'98)], IEEE, Saint-Paul (MN, USA) (15-16 May 1998).

[5] Mumtaz, R., Mumtaz, M., Mansoor, A. B., and Masood, H., "Computer aided visual inspection of aircraft surfaces," International Journal of Image Processing (IJIP) 6(1), 38-53 (2012).

[6] Malamas, E. N., Petrakis, E. G., Zervakis, M., Petit, L., and Legat, J.-D., "A survey on industrial vision systems, applications and tools," Image and Vision Computing 21(2), 171 - 188 (2003).

[7] Laws, K., "Texture energy measures," in [Proceedings of image understanding workshop], 47-51 (November 1979).

[8] Fischler, M. and Bolles, R., "Random sample consensus: A paradigm for model fitting with applications to image analysis and automated cartography," Communications of the ACM 24, 381-395 (1981).

[9] Tian, Y., Yang, X., and Arditi, A., "Computer vision-based door detection for accessibility of unfamiliar environments to blind persons," in [Proceedings of the 12th International Conference on Computers Helping People with Special Needs], ICCHP'10, 263-270, Springer-Verlag, Vienna, Austria (2010).

[10] Juenemann, C., Corbin, A., and Li, J., "Robust door detection." Final Project - Stanford Electrical Engineering Dept. Course EE368 (2010).

[11] Majumdar, J., Jain, R. P. K., M, V. G., and R, S., "Intelligent vision system for door sensing mobile robot," IAES International Journal of Robotics and Automation (IJRA) 1(4), 190-202 (2012).

[12] Muñoz Salinas, R., Aguirre, E., and García-Silvente, M., "Detection of doors using a genetic visual fuzzy system for mobile robots," Autonomous Robots 21(2), 123-141 (2006).

[13] Duda, R. O. and Hart, P. E., "Use of the hough transformation to detect lines and curves in pictures," Commun. ACM 15, 11-15 (Jan. 1972).

[14] Otsu, N., "A Threshold Selection Method from Gray-level Histograms," IEEE Transactions on Systems, Man and Cybernetics 9(1), 62-66 (1979).

[15] Xie, Y. and Ji, Q., "A new efficient ellipse detection method," in [Proceedings. 16th International Conference on Pattern Recognition], 2, 957-960, IEEE, Quebec, Canada (August 2002).

[16] Chan, T. F. and Vese, L. A., "Active contours without edges," Trans. Img. Proc. 10, 266-277 (Feb. 2001). 